\title{
Predição de cobertura em terrenos mistos de baixa rugosidade: uma abordagem via equações integrais
}

\author{
Cláudio Garcia Batista e Cássio Gonçalves do Rego
}

\begin{abstract}
Resumo-No presente trabalho é apresentada uma formulação baseada em equações integrais para propagação de ondas de rádio em terrenos suavemente irregulares não homogêneos. Assume-se a condição de contorno de Leontovich para consideração aproximada das perdas do solo e que o perfil do terreno é invariante na direção perpendicular ao plano de incidência. A formulação foi desenvolvida para polarização vertical. Para sua implementação, desenvolveu-se um software de predição concebido como ferramenta científica e acadêmica. A validade e aplicabilidade da formulação proposta foi verificada em dois estudos de caso: a cunha de Luebbers e um trabalho de medições realizado em Brasília-DF. Para o segundo caso também foi realizada a comparação com modelos empíricos (Hata) e semiempíricos (recomendação ITU-R 1546).
\end{abstract}

Palavras-Chave-Propagação radioelétrica, equações integrais, software de predição

Abstract-This work presents an integral equations formulation for radio waves propagating over inhomogeneous smoothly irregular terrain. It is assumed the Leontovich boundary condition in order to approximate the ground loss and that the terrain has no transverse variations. The formulation deals with vertical polarization. For its implementation a prediction software was produced as a scientific and academic resource. In order to validate the formulation proposed, two cases were analyzed: the wedge proposed by Luebbers and measures obtained in BrasíliaDF. For the second case it was also made a comparison with empiric models (Hata) and semi-empirics (ITU-R recommendation 1546).

Keywords - Radiowave propagation, integral equations, prediciton software.

\section{INTRODUÇÃO}

A utilização de equações integrais para análise da propagação radioelétrica vem se tornando atraente com o desenvolvimento de computadores mais eficientes e acessíveis. Apesar da maior velocidade obtida com modelos empíricos e semi-empíricos, o maior rigor da formulação através de equações integrais apresenta resultados mais coerentes.

Aproximações foram estabelecidas desde o trabalho de Hufford [1], dada a complexidade inerente das formulações propostas. Modelos baseados em equações integrais do campo elétrico (EFIE) e do campo magnético (MFIE) foram propostos por Hviid [2] e Moreira [3] para polarização vertical em terrenos suavemente irregulares. O retro-espalhamento da energia foi desprezado, permitindo o cálculo recursivo das correntes equivalentes através do Método dos Momentos

Cláudio Garcia Batista e Cássio Gonçalves do Rego, Programa de Pós-Graduação em Engenharia Elétrica, Escola de Engenharia, Universidade Federal de Minas Gerais, Belo Horizonte, e-mails: claudio@cpdee.ufmg.br,cassio@cpdee.ufmg.br. Este trabalho foi parcialmente financiado pela CAPES e pelo CEDECOM UFMG.
(MoM) evitando a resolução de sistema linear com matriz cheia. O solo foi assumido perpendicularmente invariante e o espalhamento esférico foi tratado através do método da fase estacionária. Recentemente Moreira [4] estendeu a formulação para tratamento da condutividade finita em terrenos homogêneos.

No presente trabalho é proposta uma formulação para a EFIE e MFIE considerando o terreno com condutividade finita e não homogêneo. As perdas do solo são aproximadas através da condição de contorno de Leontovich [5]. Por apresentar maior interesse prático, foi utilizada a polarização vertical no desenvolvimento da formulação. A formulação não se limita à incidência rasante. As demais aproximações, discutidas anteriormente, foram adotadas.

O software de predição radioelétrica MCNEE (Máquina para Cálculo Numérico de Espalhamento Eletromagnético) foi desenvolvido para a implementação da formulação apresentada. Ressalta-se que a preocupação é apresentar uma ferramenta didática para estudo e análise do Método dos Momentos. Resultados são apresentados para dois casos: a cunha de Luebbers [6] e o trabalho realizado por Mayrink et al. [7] com medições feitas em Brasília-DF. A solução do MCNEE apresentada para a cunha é comparada com os resultados obtidos por Moreira [4] para solo homogêneo. Para o segundo caso obteve-se as medições de campo. Os resultados do MCNEE são comparados com as medidas, modelo de HATA [8] e recomendação ITU-R 1546 [9].

O presente artigo está organizado da seguinte forma: na seção II é apresentada a formulação desenvolvida; a seção III detalha o software implementado; a seção IV exibe os resultados obtidos nos dois estudos de caso considerados; a seção V expõe as conclusões; a seção VI é dedicada a agradecimentos e finalmente a seção VII enumera as referências consultadas. Como o presente trabalho encontra-se em desenvolvimento é conveniente destacar que os resultados apresentados são preliminares e em fase de aperfeiçoamento.

\section{FORMULAÇÃO}

A. Equações integrais para correntes equivalentes em terrenos com perdas

Considerando correntes elétricas superficiais equivalentes $\overrightarrow{\mathbf{J}}_{s}(\overrightarrow{\mathbf{r}})$, e correntes magnéticas superficiais equivalentes $\overrightarrow{\mathbf{M}}_{s}(\overrightarrow{\mathbf{r}})$, a EFIE e a MFIE para o problema de campos eletromagnéticos externos a superfícies fechadas podem ser escritas, respectivamente, como [10]:

$$
\frac{\overrightarrow{\mathbf{E}}(\overrightarrow{\mathbf{r}})}{T}=\overrightarrow{\mathbf{E}}^{i}(\overrightarrow{\mathbf{r}})+Z_{0} L_{1}\left[\overrightarrow{\mathbf{J}}_{s}\left(\overrightarrow{\mathbf{r}}^{\prime}\right)\right]+L_{2}\left[\overrightarrow{\mathbf{M}}_{s}\left(\overrightarrow{\mathbf{r}}^{\prime}\right)\right],
$$




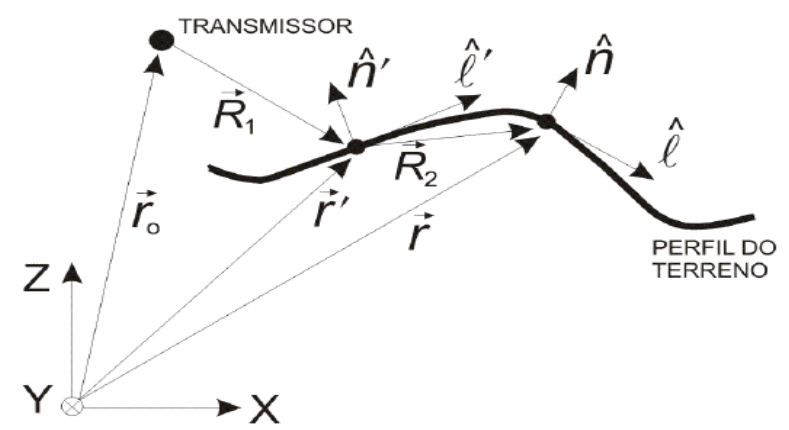

Fig. 1. Geometria básica do problema.

$$
\frac{\overrightarrow{\mathbf{H}}(\overrightarrow{\mathbf{r}})}{T}=\overrightarrow{\mathbf{H}}^{i}(\overrightarrow{\mathbf{r}})+\frac{1}{Z_{0}} L_{1}\left[\overrightarrow{\mathbf{M}}_{s}\left(\overrightarrow{\mathbf{r}}^{\prime}\right)\right]-L_{2}\left[\overrightarrow{\mathbf{J}}_{s}\left(\overrightarrow{\mathbf{r}}^{\prime}\right)\right]
$$

onde $\overrightarrow{\mathbf{E}}^{i}(\overrightarrow{\mathbf{r}})$ e $\overrightarrow{\mathbf{H}}^{i}(\overrightarrow{\mathbf{r}})$ representam os campos incidentes gerados por uma fonte transmissora, $Z_{0}$ é a impedância intrínseca do vácuo e $T$ assume o valor 1 ou 2 para o ponto de observação $\overrightarrow{\mathbf{r}}$ localizado fora ou sobre a superfície $S^{\prime}$ do terreno, respectivamente. Os operadores $L_{1}$ e $L_{2}$ aplicados a uma corrente genérica $\overrightarrow{\mathbf{I}}\left(\overrightarrow{\mathbf{r}}^{\prime}\right)$ são escritos

$$
\begin{gathered}
L_{1}\left[\overrightarrow{\mathbf{I}}\left(\overrightarrow{\mathbf{r}}^{\prime}\right)\right]=-j k_{0} \oint_{S^{\prime}}\left[\overrightarrow{\mathbf{I}}\left(\overrightarrow{\mathbf{r}}^{\prime}\right) G\left(\overrightarrow{\mathbf{r}}, \overrightarrow{\mathbf{r}}^{\prime}\right)-\frac{1}{k_{0}^{2}} \nabla^{\prime} \cdot \overrightarrow{\mathbf{I}}\left(\overrightarrow{\mathbf{r}}^{\prime}\right) \nabla^{\prime} G\left(\overrightarrow{\mathbf{r}}, \overrightarrow{\mathbf{r}}^{\prime}\right)\right] d S^{\prime} \\
L_{2}\left[\overrightarrow{\mathbf{I}}\left(\overrightarrow{\mathbf{r}}^{\prime}\right)\right]=-\oint_{S^{\prime}}\left[\overrightarrow{\mathbf{I}}\left(\overrightarrow{\mathbf{r}}^{\prime}\right) \times \nabla^{\prime} G\left(\overrightarrow{\mathbf{r}}, \overrightarrow{\mathbf{r}}^{\prime}\right)\right] d S^{\prime}
\end{gathered}
$$

onde $G\left(\overrightarrow{\mathbf{r}}, \overrightarrow{\mathbf{r}}^{\prime}\right)$ é a função de Green no espaço livre

$$
G\left(\overrightarrow{\mathbf{r}}, \overrightarrow{\mathbf{r}}^{\prime}\right)=\frac{e^{-j k_{0}\left|\overrightarrow{\mathbf{r}}-\overrightarrow{\mathbf{r}}^{\prime}\right|}}{4 \pi\left|\overrightarrow{\mathbf{r}}-\overrightarrow{\mathbf{r}}^{\prime}\right|}
$$

e $k_{0}=\omega \sqrt{\mu_{0} \epsilon_{0}}$ é a constante de propagação no vácuo. $\mathrm{O}$ vetor $\overrightarrow{\mathbf{r}^{\prime}}$ indica a posição da fonte e $\overrightarrow{\mathbf{r}}$ se refere à posição do observador.

As equações integrais apresentadas acima podem ser aplicadas para a situação ilustrada na Figura 1, na qual o terreno é representado por uma superfície aberta, invariante na direção $\hat{\mathbf{y}}$. O solo é modelado por trechos homogêneos, com diferentes parâmetros constitutivos, com perdas caracterizadas por uma impedância de superfície baseada na condição de contorno de Leontovich [5]

$$
\hat{\mathbf{n}} \times \overrightarrow{\mathbf{E}}(\mathbf{r})=Z_{g}(\overrightarrow{\mathbf{r}}) \hat{\mathbf{n}} \times[\hat{\mathbf{n}} \times \overrightarrow{\mathbf{H}}(\overrightarrow{\mathbf{r}})],
$$

onde

$$
Z_{g}(\overrightarrow{\mathbf{r}})=\frac{\sqrt{\mu_{g}(\overrightarrow{\mathbf{r}})\left[\epsilon_{g}(\overrightarrow{\mathbf{r}})-j \frac{\sigma_{g}(\overrightarrow{\mathbf{r}})}{\omega}\right]-\mu_{0} \epsilon_{0}}}{\epsilon_{g}(\overrightarrow{\mathbf{r}})-j \frac{\sigma_{g}(\overrightarrow{\mathbf{r}})}{\omega}},
$$

é uma impedância de superfície, $\hat{\mathbf{n}}$ é o vetor normal unitário ao terreno, $\mu_{g}(\overrightarrow{\mathbf{r}}), \epsilon_{g}(\overrightarrow{\mathbf{r}})$ e $\sigma_{g}(\overrightarrow{\mathbf{r}})$ são, respectivamente, a permeabilidade magnética, a permissividade elétrica e a condutividade do solo. Os parâmetros $\mu_{0}$ e $\epsilon_{0}$ são a permeabilidade e a permissividade no espaço livre. Para terrenos caracterizados pela impedância de superfície expressa em 7, a relação entre as correntes elétrica e magnética tem a forma

$$
\overrightarrow{\mathbf{J}}_{s}(\overrightarrow{\mathbf{r}})=\frac{\hat{\mathbf{n}} \times \overrightarrow{\mathbf{M}}_{s}(\overrightarrow{\mathbf{r}})}{Z_{g}(\overrightarrow{\mathbf{r}})}
$$

permitindo o emprego da formulação das equações integrais para terrenos mistos, com diferentes características eletromagnéticas.

Assume-se que as correntes equivalentes têm distribuição de fase esférica, de maneira que escreve-se [4]

$$
\begin{aligned}
\overrightarrow{\mathbf{J}}_{s}(\overrightarrow{\mathbf{r}}) & =\overrightarrow{\mathbf{J}}_{A}(\overrightarrow{\mathbf{r}}) e^{-j k_{0}\left|\overrightarrow{\mathbf{r}}-\overrightarrow{\mathbf{r}}_{0}\right|} \\
\overrightarrow{\mathbf{M}}_{s}(\overrightarrow{\mathbf{r}}) & =\overrightarrow{\mathbf{M}}_{A}(\overrightarrow{\mathbf{r}}) e^{-j k_{0}\left|\overrightarrow{\mathbf{r}}-\overrightarrow{\mathbf{r}}_{0}\right|}
\end{aligned}
$$

onde $\overrightarrow{\mathbf{J}}_{A}(\overrightarrow{\mathbf{r}})$ e $\overrightarrow{\mathbf{M}}_{A}(\overrightarrow{\mathbf{r}})$ são funções de amplitude, e o vetor $\overrightarrow{\mathbf{r}}_{0}$ localiza o ponto de transmissão dos campos incidentes, como mostrado na Figura 1.

Para a representação das correntes é adotado um sistema de coordenadas local, fixo a cada ponto do perfil do terreno $(\hat{\boldsymbol{\ell}}, \hat{\mathbf{y}}, \hat{\mathbf{n}})$ como mostrado na Figura 1. Considera-se que o terreno é invariante na direção $\hat{\mathbf{y}}$, possibilitando a aplicação do método da fase estacionária na avaliação das integrais de superfície expressas em (1) e (2). Sendo assim, podese empregar as equações (8)-(10) e reduzir as integrais de superfície que aparecem em (3) e (4) em integrais de linha em $\ell^{\prime}$, o que permite escrever

$$
\begin{gathered}
L_{1}\left[\overrightarrow{\mathbf{J}}_{s}\left(\overrightarrow{\mathbf{r}}^{\prime}\right)\right]=-k_{0} \int_{\ell^{\prime}}\left\{\frac{\hat{\mathbf{n}} \times \overrightarrow{\mathbf{M}}_{A}\left(\overrightarrow{\mathbf{r}}^{\prime}\right)}{Z_{g}\left(\overrightarrow{\mathbf{r}}^{\prime}\right)} G_{1}\left(\overrightarrow{\mathbf{r}}, \overrightarrow{\mathbf{r}}^{\prime}, \overrightarrow{\mathbf{r}}_{0}\right)-\right. \\
\left.\left[\frac{\hat{\mathbf{n}} \times \overrightarrow{\mathbf{M}}_{A}\left(\overrightarrow{\mathbf{r}}^{\prime}\right)}{Z_{g}\left(\overrightarrow{\mathbf{r}}^{\prime}\right)} \cdot \hat{\mathbf{R}}_{1}\right] \frac{\hat{\mathbf{R}}_{2}}{k_{0}} G_{2}\left(\overrightarrow{\mathbf{r}}, \overrightarrow{\mathbf{r}}^{\prime}, \overrightarrow{\mathbf{r}}_{0}\right)\right\} d \ell^{\prime}, \\
L_{2}\left[\overrightarrow{\mathbf{J}}_{s}\left(\overrightarrow{\mathbf{r}}^{\prime}\right)\right]=-k_{0} \int_{\ell^{\prime}}\left[\frac{\hat{\mathbf{n}} \times \overrightarrow{\mathbf{M}}_{A}\left(\overrightarrow{\mathbf{r}}^{\prime}\right)}{Z_{g}\left(\overrightarrow{\mathbf{r}}^{\prime}\right)} \times \hat{\mathbf{R}}_{2}\right] G_{2}\left(\overrightarrow{\mathbf{r}}, \overrightarrow{\mathbf{r}}^{\prime}, \overrightarrow{\mathbf{r}}_{0}\right) d \ell^{\prime}, \\
L_{1}\left[\overrightarrow{\mathbf{M}}_{s}\left(\overrightarrow{\mathbf{r}}^{\prime}\right)\right]=-k_{0} \int_{\ell^{\prime}} \overrightarrow{\mathbf{M}}_{A}\left(\overrightarrow{\mathbf{r}}^{\prime}\right) G_{1}\left(\overrightarrow{\mathbf{r}}, \overrightarrow{\mathbf{r}}^{\prime}, \overrightarrow{\mathbf{r}}_{0}\right) d \ell^{\prime}, \\
L_{2}\left[\overrightarrow{\mathbf{M}}_{s}\left(\overrightarrow{\mathbf{r}}^{\prime}\right)\right]=-k_{0} \int_{\ell^{\prime}}\left[\overrightarrow{\mathbf{M}}_{A}\left(\overrightarrow{\mathbf{r}}^{\prime}\right) \times \hat{\mathbf{R}}_{2}\right] G_{2}\left(\overrightarrow{\mathbf{r}}, \overrightarrow{\mathbf{r}}^{\prime}, \overrightarrow{\mathbf{r}}_{0}\right) d \ell^{\prime},
\end{gathered}
$$

onde

$$
\begin{gathered}
\mathbf{R}_{1}=\left.\left(\overrightarrow{\mathbf{r}}^{\prime}-\overrightarrow{\mathbf{r}}_{0}\right)\right|_{y^{\prime}=y_{0}}=R_{1} \hat{\mathbf{R}}_{1}= \\
=\sqrt{\left(x^{\prime}-x_{0}\right)^{2}+\left(z^{\prime}-z_{0}\right)^{2}} \hat{\mathbf{R}}_{1}, \\
\mathbf{R}_{2}=\left.\left(\overrightarrow{\mathbf{r}}-\overrightarrow{\mathbf{r}}^{\prime}\right)\right|_{y=y^{\prime}=y_{0}}=R_{2} \hat{\mathbf{R}}_{2}= \\
=\sqrt{\left(x-x^{\prime}\right)^{2}+\left(z-z^{\prime}\right)^{2}} \hat{\mathbf{R}}_{2}, \\
G_{1}\left(\overrightarrow{\mathbf{r}}, \overrightarrow{\mathbf{r}}^{\prime}, \overrightarrow{\mathbf{r}}_{0}\right)=\frac{e^{-j k_{0}\left(R_{1}+R_{2}+j \frac{\pi}{4}\right)}}{4 \pi \sqrt{\left(1+\frac{R_{1}}{R_{2}}\right) \frac{R_{2}}{\lambda_{0}}}} \\
G_{2}\left(\overrightarrow{\mathbf{r}}, \overrightarrow{\mathbf{r}}^{\prime}, \overrightarrow{\mathbf{r}}_{0}\right)=G_{1}\left(\mathbf{r}, \mathbf{r}^{\prime}, \mathbf{r}_{0}\right)\left(1+\frac{j}{k_{0} R_{2}}\right),
\end{gathered}
$$

e $\lambda_{0}$ é o comprimento de onda no espaço livre.

Observando que esse trabalho visa a polarização vertical, as correntes magnéticas equivalentes $\overrightarrow{\mathbf{M}}_{s}(\overrightarrow{\mathbf{r}})$ possuem orientação 


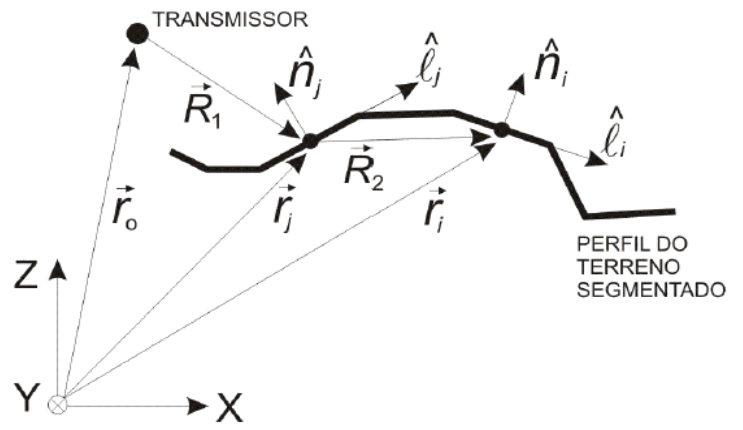

Fig. 2. Segmentação do terreno.

em $\hat{\mathbf{y}}$ e as correntes elétricas equivalentes $\overrightarrow{\mathbf{J}}_{s}(\overrightarrow{\mathbf{r}})$ em $\hat{\ell}$. Assim, as equações integrais (1) e (2) podem ser aplicadas em pontos de observação localizados sobre a superfície do terreno, de forma que é possível escrever

$$
\begin{aligned}
\hat{\mathbf{n}} \times \overrightarrow{\mathbf{E}}^{i}(\overrightarrow{\mathbf{r}})=-\frac{\overrightarrow{\mathbf{M}}_{A}(\overrightarrow{\mathbf{r}})}{2} e^{-j k_{0}\left|\overrightarrow{\mathbf{r}}-\overrightarrow{\mathbf{r}}_{0}\right|} \\
-Z_{0} k_{0} \int_{\ell^{\prime}} \frac{\overrightarrow{\mathbf{M}}_{A}\left(\overrightarrow{\mathbf{r}}^{\prime}\right)}{Z_{g}\left(\overrightarrow{\mathbf{r}}^{\prime}\right)}\left[\left(\hat{\mathbf{n}} \cdot \hat{\mathbf{n}}^{\prime}\right) G_{1}\left(\overrightarrow{\mathbf{r}}, \overrightarrow{\mathbf{r}}^{\prime}, \overrightarrow{\mathbf{r}}_{0}\right)+\right. \\
\left.\left(\hat{\ell}^{\prime} \cdot \hat{\mathbf{R}}_{1}\right)\left(\hat{\ell} \cdot \hat{\mathbf{R}}_{2}\right) G_{2}\left(\overrightarrow{\mathbf{r}}, \overrightarrow{\mathbf{r}}^{\prime}, \overrightarrow{\mathbf{r}}_{0}\right)\right] d \ell^{\prime}+ \\
\int_{\ell^{\prime}} \overrightarrow{\mathbf{M}}_{A}\left(\overrightarrow{\mathbf{r}}^{\prime}\right)\left(\hat{\mathbf{n}} \cdot \hat{\mathbf{R}}_{2}\right) G_{2}\left(\overrightarrow{\mathbf{r}}, \overrightarrow{\mathbf{r}}^{\prime}, \overrightarrow{\mathbf{r}}_{0}\right) d \ell^{\prime},
\end{aligned}
$$

$$
\begin{aligned}
\hat{\mathbf{n}} \times \overrightarrow{\mathbf{H}}^{i}(\overrightarrow{\mathbf{r}}) & =-\frac{Z_{0}}{2 Z_{g}(\overrightarrow{\mathbf{r}})} \overrightarrow{\mathbf{M}}_{A}(\overrightarrow{\mathbf{r}}) e^{-j k_{0}\left|\overrightarrow{\mathbf{r}}-\overrightarrow{\mathbf{r}}_{0}\right|} \\
& -k_{0} \int_{\ell^{\prime}} \overrightarrow{\mathbf{M}}_{A}\left(\overrightarrow{\mathbf{r}}^{\prime}\right) G_{1}\left(\overrightarrow{\mathbf{r}}, \overrightarrow{\mathbf{r}}^{\prime}, \overrightarrow{\mathbf{r}}_{0}\right) d \ell^{\prime}+ \\
& Z_{0} k_{0} \int_{\ell^{\prime}} \frac{\overrightarrow{\mathbf{M}}_{A}\left(\overrightarrow{\mathbf{r}}^{\prime}\right)}{Z_{g}\left(\overrightarrow{\mathbf{r}}^{\prime}\right)}\left(\mathbf{n}^{\prime} \cdot \hat{\mathbf{R}}_{2}\right) G_{2}\left(\overrightarrow{\mathbf{r}}, \overrightarrow{\mathbf{r}}^{\prime}, \overrightarrow{\mathbf{r}}_{0}\right) d \ell^{\prime} .
\end{aligned}
$$

\section{B. Método dos Momentos}

As correntes equivalentes $\overrightarrow{\mathbf{J}}_{s}(\overrightarrow{\mathbf{r}})$ e $\overrightarrow{\mathbf{M}}_{s}(\overrightarrow{\mathbf{r}})$ podem ser determinadas a partir da aplicação do método dos momentos, que consiste na segmentação do perfil do terreno como indicado na Figura 2, na expansão de $\overrightarrow{\mathbf{M}}_{A}(\overrightarrow{\mathbf{r}})$ na forma de uma combinação linear de funções de base $\overrightarrow{\mathbf{m}}_{j}(\overrightarrow{\mathbf{r}})$ com amplitudes complexas $\mathbf{M}_{\mathbf{j}}$

$$
\overrightarrow{\mathbf{M}}_{A}(\overrightarrow{\mathbf{r}})=\sum_{j=1}^{N_{b}} M_{j} \overrightarrow{\mathbf{m}}_{j}(\overrightarrow{\mathbf{r}}),
$$

e na aplicação de funções de peso $\overrightarrow{\mathbf{w}}_{i}(\mathbf{r})$ em (19) e (20). Para a função de base utilizou-se um pulso unitário com orientação em $\hat{\mathbf{y}}$. Foi utilizada o Delta de Dirac como função de peso com orientação em $\hat{\mathbf{y}}$ para a EFIE e em $\hat{\ell}$ para a MFIE [2].
Assim, se escreve

$$
\begin{aligned}
& \int_{\ell} \overrightarrow{\mathbf{w}}_{i}(\overrightarrow{\mathbf{r}}) \cdot\left[\hat{\mathbf{n}} \times \overrightarrow{\mathbf{E}}^{i}(\overrightarrow{\mathbf{r}})\right] d \ell= \\
& \quad-\frac{1}{2} \sum_{j=1}^{N} M_{j} \int_{\ell} \overrightarrow{\mathbf{w}}_{i}(\overrightarrow{\mathbf{r}}) \cdot \overrightarrow{\mathbf{m}}_{j}(\overrightarrow{\mathbf{r}}) e^{-j k_{0}\left|\overrightarrow{\mathbf{r}}-\overrightarrow{\mathbf{r}}_{0}\right|} d \ell \\
& -Z_{0} k_{0} \sum_{j=1}^{N} M_{j} \int_{\ell} \int_{\ell^{\prime}} \frac{\overrightarrow{\mathbf{w}}_{i}(\overrightarrow{\mathbf{r}}) \cdot \overrightarrow{\mathbf{m}}_{j}\left(\overrightarrow{\mathbf{r}}^{\prime}\right)}{Z_{g}\left(\overrightarrow{\mathbf{r}}^{\prime}\right)}\left[\left(\hat{\mathbf{n}} \cdot \hat{\mathbf{n}}^{\prime}\right) G_{1}\left(\overrightarrow{\mathbf{r}}, \overrightarrow{\mathbf{r}}^{\prime}, \overrightarrow{\mathbf{r}}_{0}\right)+\right. \\
& \left.\quad\left(\hat{\ell}^{\prime} \cdot \hat{\mathbf{R}}_{1}\right)\left(\hat{\ell} \cdot \hat{\mathbf{R}}_{2}\right) G_{2}\left(\overrightarrow{\mathbf{r}}, \overrightarrow{\mathbf{r}}^{\prime}, \overrightarrow{\mathbf{r}}_{0}\right)\right] d \ell^{\prime} d \ell+ \\
& \sum_{j=1}^{N} M_{j} \int_{\ell} \int_{\ell^{\prime}} \overrightarrow{\mathbf{w}}_{i}(\overrightarrow{\mathbf{r}}) \cdot \overrightarrow{\mathbf{m}}_{j}\left(\overrightarrow{\mathbf{r}}^{\prime}\right)\left(\hat{\mathbf{n}} \cdot \hat{\mathbf{R}}_{2}\right) G_{2}\left(\overrightarrow{\mathbf{r}}, \overrightarrow{\mathbf{r}}^{\prime}, \overrightarrow{\mathbf{r}}_{0}\right) d \ell^{\prime} d \ell,
\end{aligned}
$$

$$
\begin{gathered}
\int_{\ell} \overrightarrow{\mathbf{w}}_{i}(\overrightarrow{\mathbf{r}}) \cdot\left[\hat{\mathbf{n}} \times \overrightarrow{\mathbf{H}}^{i}(\overrightarrow{\mathbf{r}})\right] d \ell= \\
\quad-\frac{Z_{0}}{2} \sum_{j=1}^{N} M_{j} \int_{\ell} \frac{\overrightarrow{\mathbf{w}}_{i}(\overrightarrow{\mathbf{r}}) \cdot \mathbf{m}_{j}(\overrightarrow{\mathbf{r}})}{Z_{g}(\overrightarrow{\mathbf{r}})} e^{-j k_{0}\left|\overrightarrow{\mathbf{r}}-\overrightarrow{\mathbf{r}}_{0}\right|} d \ell \\
k_{0} \sum_{j=1}^{N} M_{j} \int_{\ell} \int_{\ell^{\prime}} \overrightarrow{\mathbf{w}}_{i}(\overrightarrow{\mathbf{r}}) \cdot \mathbf{m}_{j}\left(\overrightarrow{\mathbf{r}}^{\prime}\right) G_{1}\left(\overrightarrow{\mathbf{r}}, \overrightarrow{\mathbf{r}}^{\prime}, \overrightarrow{\mathbf{r}}_{0}\right) d \ell^{\prime} d \ell+ \\
Z_{0} k_{0} \sum_{j=1}^{N} M_{j} \int_{\ell} \int_{\ell^{\prime}} \frac{\overrightarrow{\mathbf{w}}_{i}(\overrightarrow{\mathbf{r}}) \cdot \overrightarrow{\mathbf{m}}_{j}\left(\overrightarrow{\mathbf{r}}^{\prime}\right)}{Z_{g}\left(\overrightarrow{\mathbf{r}}^{\prime}\right)}\left(\hat{\mathbf{n}}^{\prime} \cdot \hat{\mathbf{R}}_{2}\right) G_{2}\left(\overrightarrow{\mathbf{r}}, \overrightarrow{\mathbf{r}}^{\prime}, \overrightarrow{\mathbf{r}}_{0}\right) d \ell^{\prime} d \ell
\end{gathered}
$$

As integrais em $\ell^{\prime}$ foram resolvidas considerando, para a fase do integrando, $\mathbf{R}_{1}$ e $\mathbf{R}_{1}$ variando linearmente ao longo do segmento [11], enquanto que a amplitude do integrando é tomada no ponto médio de cada segmento. Moreira em [4] considera $\mathbf{R}_{1}$ e $\mathbf{R}_{1}$ constantes ao longo do segmento. Assim, para a EFIE:

$$
V_{i}=\overrightarrow{\mathbf{E}}^{i}(\overrightarrow{\mathbf{r}}) \cdot \hat{\ell}
$$

$$
\begin{gathered}
Z_{i j}=\frac{\sin (\alpha)}{\alpha} K \Delta_{j}\left\{\left(\hat{\mathbf{n}}_{i} \cdot \hat{\mathbf{R}}_{2}\right) G_{2}\left(\overrightarrow{\mathbf{r}}, \overrightarrow{\mathbf{r}}^{\prime}, \overrightarrow{\mathbf{r}}_{0}\right)\right. \\
\left.-\frac{Z_{0}}{Z_{g}(\overrightarrow{\mathbf{r}})}\left[\left(\hat{\mathbf{n}}_{i} \cdot \hat{\mathbf{n}}_{j}\right) G_{1}\left(\overrightarrow{\mathbf{r}}, \overrightarrow{\mathbf{r}}^{\prime}, \overrightarrow{\mathbf{r}}_{0}\right)+\left(\hat{\boldsymbol{\ell}}_{j} \cdot \hat{\mathbf{R}}_{1}\right)\left(\hat{\ell}_{i} \cdot \hat{\mathbf{R}}_{2}\right) G_{2}\left(\overrightarrow{\mathbf{r}}, \overrightarrow{\mathbf{r}}^{\prime}, \overrightarrow{\mathbf{r}}_{0}\right)\right]\right\}
\end{gathered}
$$

$$
\begin{gathered}
Z_{i i}=-\frac{e^{-j k_{0}\left|\overrightarrow{\mathbf{r}}-\overrightarrow{\mathbf{r}}_{0}\right|}}{2}\{1+ \\
\left.\frac{Z_{0}}{Z_{g}(\overrightarrow{\mathbf{r}})} e^{j \frac{\pi}{4}}\left[\operatorname{Fresnel}\left(\frac{\sqrt{A_{1} K \Delta_{j} / \pi}}{\sqrt{A_{1}}}\right)+\operatorname{Fresnel}\left(\frac{\sqrt{A_{2} K \Delta_{j} / \pi}}{\sqrt{A_{2}}}\right)\right]\right\}
\end{gathered}
$$

e para a MFIE:

$$
V_{i}=Z_{0}\left[\overrightarrow{\mathbf{H}}^{i}(\overrightarrow{\mathbf{r}}) \cdot \hat{\mathbf{y}}\right]
$$




$$
\begin{aligned}
& Z_{i j}=\frac{\sin (\alpha)}{\alpha} K \Delta_{j} {\left[G_{1}\left(\overrightarrow{\mathbf{r}}, \overrightarrow{\mathbf{r}}^{\prime}, \overrightarrow{\mathbf{r}}_{0}\right)\right.} \\
&\left.\left.-\frac{Z_{0}}{Z_{g}(\overrightarrow{\mathbf{r}})}\left(\hat{\mathbf{n}}_{j} \cdot \hat{\mathbf{R}}_{2}\right) G_{2}\left(\overrightarrow{\mathbf{r}}, \overrightarrow{\mathbf{r}}^{\prime}, \overrightarrow{\mathbf{r}}_{0}\right)\right)\right] \quad(28) \\
& Z_{i i}=\frac{e^{-j k_{0}\left|\overrightarrow{\mathbf{r}}-\overrightarrow{\mathbf{r}}_{0}\right|}}{2}\left\{\frac{Z_{0}}{Z_{g}(\overrightarrow{\mathbf{r}})}+\right. \\
&\left.e^{j \frac{\pi}{4}}\left[\operatorname{Fresnel}\left(\frac{\sqrt{A_{1} K \Delta_{j} / \pi}}{\sqrt{A_{1}}}\right)+\operatorname{Fresnel}\left(\frac{\sqrt{A_{2} K \Delta_{j} / \pi}}{\sqrt{A_{2}}}\right)\right]\right\}
\end{aligned}
$$

onde:

$$
\begin{gathered}
\alpha=\frac{K \Delta_{j}}{2}\left(\hat{\mathbf{R}}_{1}-\hat{\mathbf{R}}_{2}\right) \cdot \hat{\boldsymbol{\ell}}_{j} \\
A_{1}=1-\left(\hat{\mathbf{R}}_{1} \cdot \hat{\boldsymbol{\ell}}_{j}\right) \\
A_{2}=1+\left(\hat{\mathbf{R}}_{1} \cdot \hat{\boldsymbol{\ell}}_{j}\right)
\end{gathered}
$$

$\Delta_{j}$ é o comprimento do segmento je Fresnel $(x)$ é definido pelas integrais de Fresnel $C(x)$ e $S(x)$ como:

$$
\text { Fresnel }(x)=C(x)-j S(x)
$$

Finalmente, as correntes $M_{j}$ são calculadas da maneira usual através do sistema linear $\left[V_{i}\right]=\left[Z_{i j}\right]\left[M_{j}\right]$. Como o terreno foi assumido eletricamente suave é possível descartar o retro-epalhamento e calcular as correntes de maneira recursiva, evitando a solução do sistema linear com matriz $\left[Z_{i j}\right]$ cheia. Assim:

$$
M_{j}=\frac{1}{Z_{i} j}\left[V_{i}-\sum_{j=1}^{i-1} Z_{i j} M_{j}\right]
$$

Uma vez obtidas as correntes equivalentes o campo espalhado pode ser calculado através de (1), (2) e (8), observando que agora $T=1$.

\section{Software de Predição de Propagação}

Objetivando a implementação das equações propostas, está sendodesenvolvido um software de predição de propagação denominado MCNEE (Máquina para Cálculo Numérico de Espalhamento Eletromagnético), utilizando compilador Visual Fortran 9.1 (c) para elaboração das rotinas de cálculo, e pacote Visual Studio 2005 (c) para a interface gráfica. O MCNEE também permite comparações com outros modelos (empíricos e semi-empíricos) e tem preocupação em se tornar uma ferramenta didática para os meios Acadêmico e Científico.

Em virtude do software estar ainda inacabado, no momento, o cálculo do espalhamento eletromagnético é restrito a terrenos eletricamente suaves e a faixas de freqüência ente VHF e UHF.

Através da interface gráfica é possível selecionar o perfil do terreno e organizá-lo em intervalos com características elétricas distintas. Configurações padrões de solos utilizadas

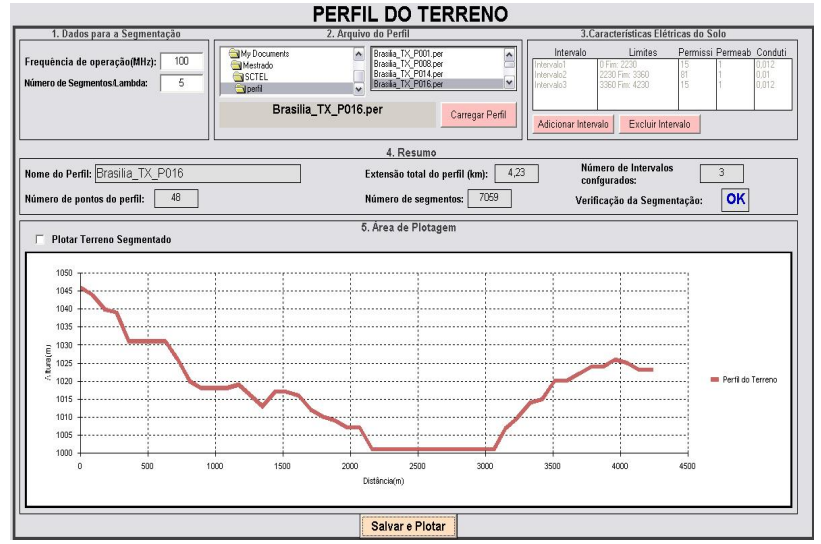

Fig. 3. Tela de configuração do terreno do MCNEE.

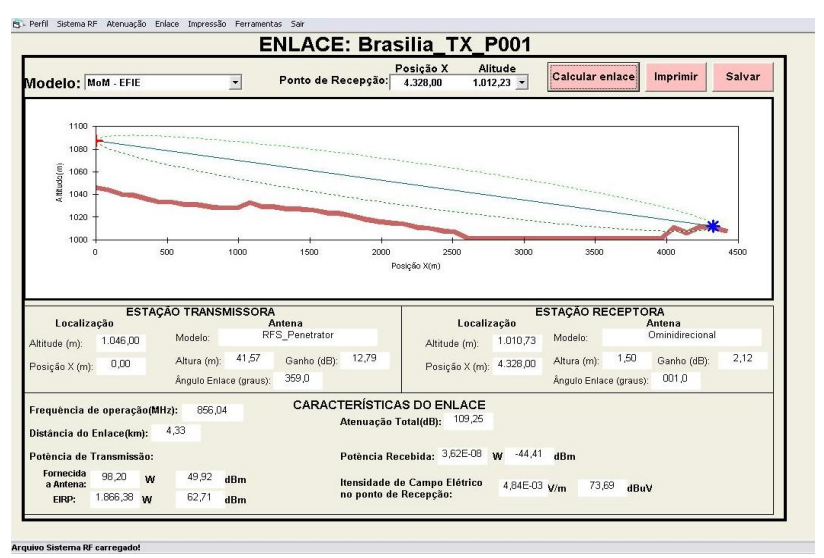

Fig. 4. Tela de cálulo de Enlace do MCNEE.

na literatura [12] são disponíveis para o usuário estimar o comportamento elétrico do terreno.

O MCNEE permite a configuração dos principais parâmetros do sistema de radiofreqüência das estações Transmissora e Receptora. Modelos de antenas diversos podem ser utilizados, se fornecidos seus respectivos diagramas de irradiação. A posição da Estação Receptora pode sofrer variações ao longo do terreno e a atenuação total computada através do modelo de cálculo selecionado. A tela de Cálculo de Enlace exibe as principais características do perfil analisado, bem como os parâmetros calculados do enlace. Os enlaces podem ser salvos em um arquivo de banco de dados.

\section{Resultados}

\section{A. Cunha de Luebbers}

A atenuação resultante da cunha proposta por Luebbers [6] é analisada conforme a Figura $5 \mathrm{com} h=50 \mathrm{~m}$. O dipolo de Hertz foi utilizado como transmissor com orientação vertical irradiando em $100 \mathrm{MHz}$. O dipolo é posicionado no início da cunha com altura de $10 \mathrm{~m}$. A atenuação total foi calculada para duas situações: variação da altura $h_{R}$ do receptor posicionado no final da cunha $\left(d_{R}=5 \mathrm{~km}\right)$ e variação da posição $d_{R}$ do receptor com altura $h_{R}=1 \mathrm{~m}$.

A figura 6 apresenta os resultados da atenuação pela cunha produzidos pelo MCNEE comparados com a solução da UTD 


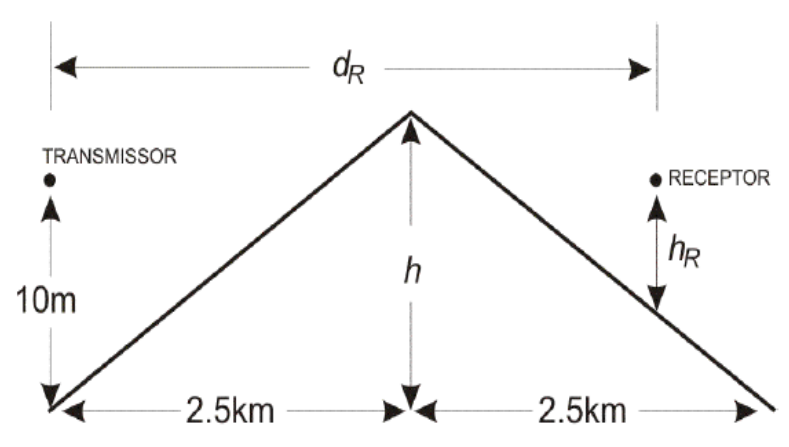

Fig. 5. Cunha utilizada na simulação.

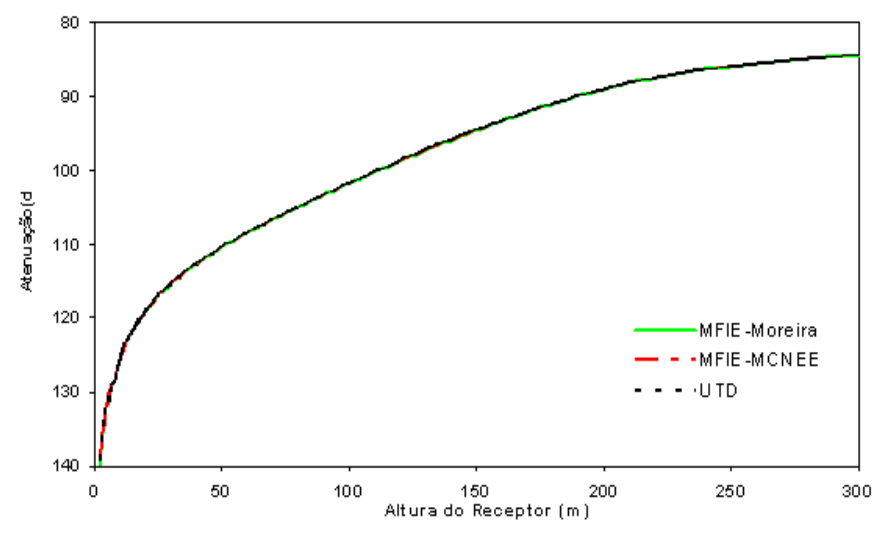

Fig. 6. Atenuação em função da altura do receptor h R em $100 \mathrm{MHz}$.

heurística de Holm [13] e da MoM-MFIE de Moreira [4]. Observa-se que Moreira considera os valores de $\mathbf{R}_{1}$ e $\mathbf{R}_{1}$ constantes na fase do integrando em [4]. Conseqüentemente, foi necessário um maior número de segmentos por lambda para a convergência com a referência [13] do que o MCNEE. Para o cálculo do Método dos Momentos foi utilizado 4 segmentos $/ \lambda$ e o terreno caracterizado por $\epsilon_{g}=15 \epsilon_{0}$ e $\sigma_{g}=0,012$ $\mathrm{S} / \mathrm{m}$.

\section{B. Medições Realizadas em Brasília-DF}

Nesta seção, é analisado um caso de estudo realizado nas proximidades do Lago Paranoá, em Brasília-DF (Figura 8). As medições foram realizadas por Mayrink et al. [7] em 166 pontos ao longo do lago, resultando em enlaces somente sobre terra e enlaces sobre terra-água (terreno misto).

As medições foram realizadas em $856.039 \mathrm{MHz}$ com a estação transmissora utilizando antena modelo RFS Penetrator BRM6 (8,14 dB ganho), altura de $30 \mathrm{~m}$ e com potência de saída de $100 \mathrm{~W}$. Na recepção, utilizou-se antena omnidirecional ASP 1897T (3 dB ganho) com altura de 1,5 m.

Para a obtenção dos resultados numéricos desse artigo, optou-se por escolher 25 enlaces ao acaso percorrendo todo o entorno do lago. Os respectivos perfis foram obtidos através do Sistema de Informações Geográficas da ANATEL com uma resolução de $90 \mathrm{~m}$. Observa-se que o cálculo através do Método dos Momentos envolve a expressão de campo incidente gerado pela fonte e não foi possível obtenção de

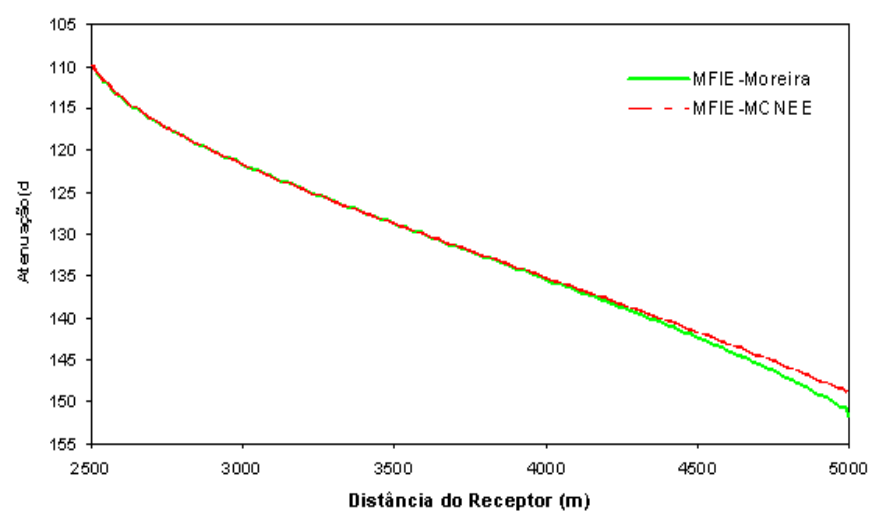

Fig. 7. Atenuação em função da altura do receptor h R em $100 \mathrm{MHz}$.

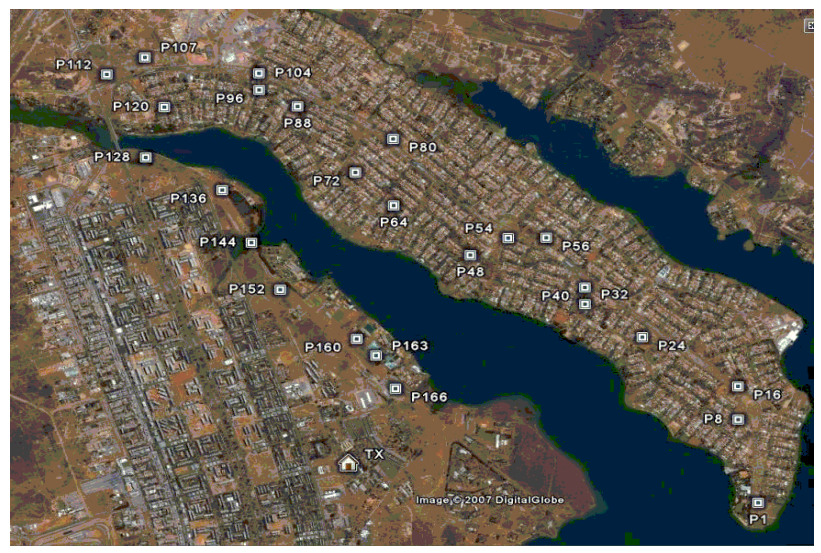

Fig. 8. Mapa da localização das medições.

tais informações para a antena utilizada nos testes. Assim, o campo gerado pela antena transmissora foi aproximado como campo distante e seu comportamento espacial foi estimado através do diagrama de irradiação vertical da antena. Para a geração dos resultados, utilizou-se uma relação de 5 segmentos por comprimento de onda e as seguintes configurações de solo: $\epsilon_{g}=15 \epsilon_{0}$ e $\sigma_{g}=0,012 \mathrm{~S} / \mathrm{m}$ para terra e $\epsilon_{g}=81 \epsilon_{0}, \sigma_{g}=0,01$ $\mathrm{S} / \mathrm{m}$ para água doce [12].

Na Figura 9, as medidas da atenuação dos enlaces são comparadas com os resultados do MoM-MFIE, MoM-EFIE e modelo de HATA gerados pelo MCNEE. Também são exibidos os resultados obtidos por Mayrink [7], utilizando a recomendação ITU-R 1546.

Os valores absolutos das diferenças (erro absoluto) entre as medidas e os modelos utilizados são mostrados para cada enlace na Figura 10.

As médias dos erros absolutos dos 25 enlaces são mostrados na Tabela I, com o Método dos Momentos apresentado menores valores. Ressalta-se que a precisão do MoM seria significativamente melhor caso se conhece-se a expressão do campo irradiado pela antena transmissora e mapas com melhor resolução fossem utilizados para obtenção dos perfis. 


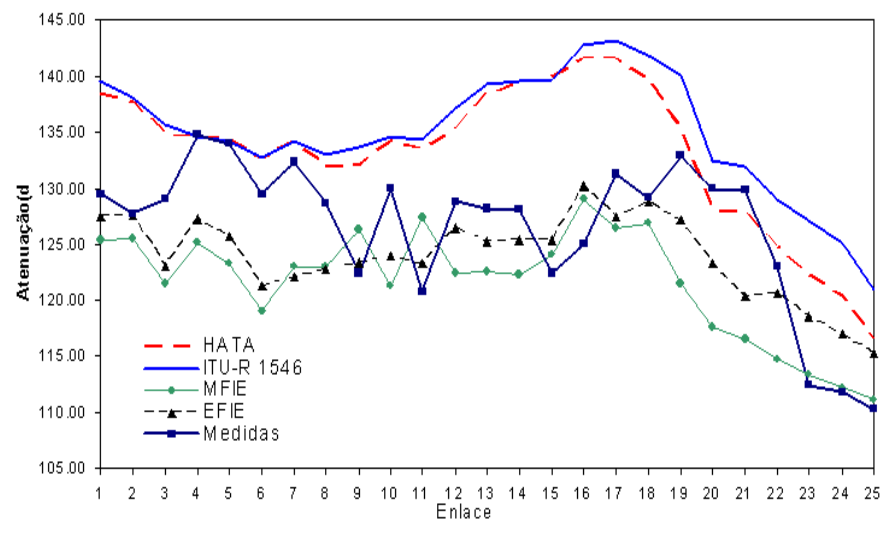

Fig. 9. Comparação da atenuação (dB) Medida, MoM-MFIE, MoM-EFIE, HATA e ITU-R 154.

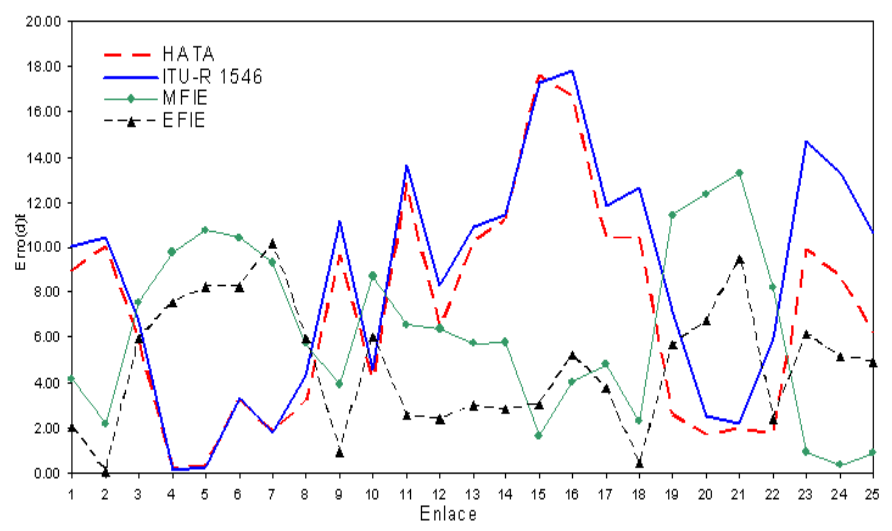

Fig. 10. Erro absoluto (dB) entre a MoM-MFIE, MoM-EFIE, HATA, ITUR1546 e as medidas realizadas.

\section{CONClusões}

Este artigo apresentou formulações para EFIE e MFIE para propagação em terrenos eletricamente suaves não homogêneos. O resultado é uma extensão de [3], [4] e [11] para terrenos mistos apresentando convergência mais rápida para o mesmo número de segmentos/ $\lambda$. Foi utilizada a polarização vertical e o modelo é válido para ondas eletromagnéticas na faixa de VHF e UHF.

O software MCNEE (Máquina para Cálculo Numérico de Espalhamento Eletromagnético) é apresentado como ferramenta didática para estudo e análise do Método dos Momentos. Como o software ainda se encontra em desenvolvimento, deseja-se estender sua aplicação para terrenos com alta rugosidade e para as faixas entre MF e HF.

TABELA I

ERRO ABSOLUTO MÉDIO DOS MODELOS ANALISADOS.

\begin{tabular}{|c|c|}
\hline Modelo de Cálculo & Erro Médio (dB) \\
\hline \hline Hata & 7,04 \\
\hline ITU-R 1546 & 8,52 \\
\hline MoM-MFIE & 6,28 \\
\hline MoM-EFIE & 4,75 \\
\hline
\end{tabular}

Estudos de casos foram realizados para validação do trabalho. A simulação utilizando a cunha apresentou resultados satisfatórios e convergência mais rápida que [4]. No caso de Brasília-DF o MCNEE calculou valores mais próximos às medições aferidas do que os modelos de Hata [8] e a recomendação ITU-R 1546 [9]. Resultados melhores poderiam ser obtidos caso a expressão de campo irradiado pela antena fosse conhecida e mapas com melhor resolução estivessem acessíveis.

\section{AGRADECIMENTOS}

Os autores gostariam de agradecer ao Prof. Antônio José Martins Soares, Eng. Elmo V. Melo, Eng. Marco Antônio de Souza Mayrink, Prof. Paulo Henrique Portela de Carvalho e Prof. Fernando José da Silva Moreira pela gentileza de disponibilizar os dados das medições realizadas em BrasíliaDF [7].

\section{REFERENNCIAS}

[1] G. A. Hufford, "An integral equation approach to the problem of wavepropagation over an irregular surface", Quarterly Appl. Math., vol.9, pp.391-404, Jan. 1952.

[2] J. T. Hviid, J. B. Andersen, J. Toftgård, and J. Bøjer, "Terrainbasedpropagation model for rural area-An integral equation approach", IEEE Trans. Antennas Propagat., vol.43, pp.41-46, Jan. 1995.

[3] F. J. S. Moreira, "MFIE-based propagation prediction", SBMO/IEEE MTT-S Conf. Proc., pp. 199-202, Aug. 2001.

[4] F. J. S. Moreira, "Aplicação de Equações Integrais para a Predição da Propagação Radioelétrica sobre Solos Suavemente Irregulares e Incidência Rasante", X Simpósio Brasileiro de Microondas e Optoeletrônica (SBMO 2002), Recife, PE, pp.191-195, Aug. 2002.

[5] T. B. A. Senior and J. L. Volakis, Approximate Boundary Conditions inElectromagnetics. London, U.K.: IEE, 1995, ch. 2.

[6] R. J. Luebbers, "Finite conductivity uniform GTD versus knife edge diffraction in prediction of propagation path loss", IEEE Trans. Antennas Propagat., vol. AP-32, pp. 70-76, Jan. 1984.

[7] A. J. M. Soares, C. G. Rego, E. V. Melo, F. J. S. Moreira, G. W. O. Costa, J. P. D. M. Pinto, M. A. S. Mayrink, N. Pasquali, P. H. P. Carvalho, and W. K. Guimarães, "Measurements of a CW Signal in Brazil and Comparison with Prediction using ITU-R P.1546-1“, 2005 IEEE/ACES International Conference on Wireless Communications and Applied Computational Electromagnetics, Hawaii, USA, pp.824-827, Apr. 2005.

[8] M. Hata, "Empirical formulae for propagation loss in land mobile radio services", IEEE Transact. Vehicular Technology, vol. VT-29, pp.317325, 1980.

[9] International Communication Union, "ITU-R P.1546-2 - Method for point-to-area for terrestrial services in the frequency range $30 \mathrm{MHz}$ to $3000 \mathrm{MHz} "$, ITU Recommendation, Agosto 2005.

[10] A. J. Poggio and E. K. Miller, Computer Techniques for Electromagnetics.Oxford, U.K.: Pergamon, 1973, ch. 4.

[11] R. B. V. Teperino e F. J. S. Moreira; "Aplicação do Método FAFFA para a Predição de Propagação sobre Terrenos Suavemente Irregulares", V Congresso Brasileiro de Eletromagnetismo (CBMag 2002), Gramado, RS, Nov. 2002.

[12] C. A. Balanis, Advanced Engineering Electromagnetics, John Wiley and Sons, 1989, ch 2-4.

[13] P. D. Holm, "A new heuristic UTD diffraction coefficient for nonperfectly conducting wedges", IEEE Trans. Antennas Propagat., vol.48, pp.1211-1219, Aug. 2000.

[14] H. Gesny, 0. Ravar, "Propagation Over Irregular Terrain In The Vhf Band : A Review Of Integral Equation Models", National Conference on Antennas and Propagation: 30 March - l April 1999 , Conference Publication No. 461,IEE, 1999. 